PROCEEDINGS OF THE

AMERICAN MATHEMATICAL SOCIETY

Volume 128, Number 11, Pages 3153-3157

S 0002-9939(00)05381-8

Article electronically published on May 2, 2000

\title{
ON THE SYMMETRY OF THE GOLDIE AND CS CONDITIONS FOR PRIME RINGS
}

\author{
DINH VAN HUYNH, S. K. JAIN, AND S. R. LÓPEZ-PERMOUTH
}

(Communicated by Ken Goodearl)

\begin{abstract}
It is shown that: (a) If $R$ is a prime right Goldie right CS ring with right uniform dimension at least 2, then $R$ is left Goldie, left CS; (b) A semiprime ring $R$ is right Goldie left CS iff $R$ is left Goldie, right CS.
\end{abstract}

All rings are associative having an identity and all modules are unitary. A right module $M$ over a ring $R$ is called CS (or extending) if every submodule of $M$ is essential in a direct summand of $M$, or equivalently, if every complement submodule of $M$ is a direct summand of $M$. A ring $R$ is called right CS (resp., left CS), if $R_{R}$ (resp., ${ }_{R} R$ ) is a CS module. CS modules have been extensively studied by many authors.

A ring $R$ is defined to be a right (left) Goldie ring if $R$ has ascending chain condition on right (left) annihilators and the right (left) uniform dimension of $R$ is finite. A right Goldie ring $R$ is (semi-)prime if and only if $R$ has classical right quotient ring which is (semi-)simple artinian. For notation not defined here we refer the reader to [1], [2] and [3].

Theorem 1. A prime right Goldie, right $C S$ ring $R$ with right uniform dimension at least 2, is left Goldie, and left CS.

Proof. Let $n$ be the right uniform dimension of $R$. By assumption, $n \geq 2$. Since $R$ is right CS, $R=e_{1} R \oplus \cdots \oplus e_{n} R$ where each $e_{i} R$ is uniform and $\left\{e_{i}\right\}_{i=1}^{n}$ is a system of orthogonal idempotents of $R$. Let $Q$ be the classical right quotient ring of $R$. Then we have:

$$
R \cong\left(\begin{array}{cccc}
e_{1} R e_{1} & e_{1} R e_{2} & \cdots & e_{1} R e_{n} \\
e_{2} R e_{1} & e_{2} R e_{2} & \cdots & e_{2} R e_{n} \\
\cdot & \cdot & \cdots & \cdot \\
\cdot & \cdot & \cdots & \cdot \\
\cdot & \cdot & \cdots & \cdot \\
e_{n} R e_{1} & e_{n} R e_{2} & \cdots & e_{n} R e_{n}
\end{array}\right) \subseteq\left(\begin{array}{cccc}
e_{1} Q e_{1} & e_{1} Q e_{2} & \cdots & e_{1} Q e_{n} \\
e_{2} Q e_{1} & e_{2} Q e_{2} & \cdots & e_{2} Q e_{n} \\
\cdot & \cdot & \cdots & \cdot \\
\cdot & \cdot & \cdots & \cdot \\
\cdot & \cdot & \cdots & \cdot \\
e_{n} Q e_{1} & e_{n} Q e_{2} & \cdots & e_{n} Q e_{n}
\end{array}\right) \cong Q
$$

Received by the editors May 12, 1998 and, in revised form, September 28, 1998 and December 9, 1998.

1991 Mathematics Subject Classification. Primary 16P60, 16N60, 16D80. 
Henceforth, we will identify

$$
\left(\begin{array}{cccc}
e_{1} R e_{1} & e_{1} R e_{2} & \cdots & e_{1} R e_{n} \\
e_{2} R e_{1} & e_{2} R e_{2} & \cdots & e_{2} R e_{n} \\
\cdot & \cdot & \cdots & \cdot \\
\cdot & \cdot & \cdots & \cdot \\
e_{n} R e_{1} & e_{n} R e_{2} & \cdots & e_{n} R e_{n}
\end{array}\right)
$$

with $R$ and

$$
\left(\begin{array}{cccc}
e_{1} Q e_{1} & e_{1} Q e_{2} & \cdots & e_{1} Q e_{n} \\
e_{2} Q e_{1} & e_{2} Q e_{2} & \cdots & e_{2} Q e_{n} \\
\cdot & \cdot & \cdots & \cdot \\
\cdot & \cdot & \cdots & \cdot \\
\cdot & \cdot & \cdots & \cdot \\
e_{n} Q e_{1} & e_{n} Q e_{2} & \cdots & e_{n} Q e_{n}
\end{array}\right)
$$

with $Q$.

Let $\alpha=\left(\begin{array}{ccc}a_{1} & \cdots & . \\ a_{2} & \cdots & . \\ \cdot & \cdots & . \\ . & \cdots & . \\ \cdot & \cdots & . \\ a_{n} & \cdots & .\end{array}\right)$ be an element of $Q$ with $a_{i} \in e_{i} Q e_{1}, \quad a_{1} \neq 0$. Then

for the minimal right ideal $M=\left(\begin{array}{cccc}e_{1} Q e_{1} & e_{1} Q e_{2} & \cdots & e_{1} Q e_{n} \\ 0 & 0 & \cdots & 0 \\ \cdot & \cdot & \cdots & \cdot \\ \cdot & \cdot & \cdots & . \\ . & . & \cdots & . \\ 0 & 0 & \cdots & 0\end{array}\right)$ of $Q, \alpha M$ is

a minimal right ideal of $Q$, too. Hence $R \cap \alpha M$ is a (nonzero) closed uniform right ideal of $R$. Consequently, $R \cap \alpha M$ is generated by an idempotent $e \in R$.

Therefore, there exists an element $\beta=\left(\begin{array}{cccc}x_{1} & x_{2} & \cdots & x_{n} \\ 0 & 0 & \cdots & 0 \\ . & . & \cdots & . \\ . & . & \cdots & . \\ . & . & \cdots & . \\ 0 & 0 & \cdots & 0\end{array}\right)$ such that $\alpha \beta=$ $\left(\begin{array}{cccc}a_{1} x_{1} & a_{1} x_{2} & \cdots & a_{1} x_{n} \\ a_{2} x_{1} & a_{2} x_{2} & \cdots & a_{2} x_{n} \\ \cdot & \cdot & \cdots & \cdot \\ \cdot & \cdot & \cdots & \cdot \\ \cdot & \cdot & \cdots & \cdot \\ a_{n} x_{1} & a_{n} x_{2} & \cdots & a_{n} x_{n}\end{array}\right)=e \in R$, where $x_{i} \in e_{1} Q e_{i}$. Hence $a_{i} x_{j} \in e_{i} R e_{j}$ for $i, j=1, \cdots, n$. Note that at least one $x_{i}$ is nonzero. After squaring this matrix and comparing the corresponding entries of this matrix and its square we get $\left(a_{1} x_{k}\right)\left(a_{k} x_{k}\right)+\cdots+\left(a_{1} x_{n}\right)\left(a_{n} x_{k}\right)=a_{1} x_{k}$, where $x_{k}$ is the first nonzero entry in the first row of $\beta$. Since $a_{1} \neq 0$, it follows $x_{k} a_{k} x_{k}+x_{k+1} a_{k+1} x_{k}+\cdots+x_{n} a_{n} x_{k}=x_{k}$. As $x_{k} \in e_{1} Q e_{k}$, there exists $x_{k}^{*} \in e_{k} Q e_{1}$ such that $x_{k} x_{k}^{*}=e_{1}$, because $e_{1} Q \cong e_{k} Q$. 
Consequently,

$$
x_{k} a_{k}+x_{k+1} a_{k+1}+\cdots+x_{n} a_{n}=e_{1} .
$$

Note that if $e_{1} R e_{1}$ is a division ring, then $R$ is a simple artinian ring. In this case the statement in our theorem is trivially true. Therefore, we assume that $e_{1} R e_{1} \neq e_{1} Q e_{1}$. Since $n>1$, we can choose $a_{1} \in e_{1} Q e_{1} \backslash e_{1} R e_{1}, a_{2}=\cdots=$ $a_{n-1}=0,0 \neq a_{n} \in e_{n} R e_{1}$. Then we must have $k=1$, i.e. $x_{1} \neq 0$. For, if $x_{1}=0$, the equation (1) becomes $x_{n} a_{n}=e_{1}$. Multiplying this with $a_{1}$ on the left we get $\left(a_{1} x_{n}\right) a_{n}=a_{1} e_{1}=a_{1}$. From this and $a_{1} x_{n} \in e_{1} R e_{n}$, it follows that $a_{1} \in e_{1} R e_{1}$, a contradiction. Hence $x_{1} \neq 0$. Thus equation (1) becomes $x_{1} a_{1}+x_{n} a_{n}=e_{1}$, and so

$$
x_{1} a_{1}=e_{1}-x_{n} a_{n} .
$$

Let $0 \neq y \in e_{1} R e_{n} \subset e_{1} Q e_{n}$. So there exists $y^{\prime} \in e_{n} Q e_{1}$, such that $y^{\prime} y=e_{n}$, because $e_{1} Q \cong e_{n} Q$. We note that $y a_{n} \neq 0$. For, if $y a_{n}=0$, then $y^{\prime}\left(y a_{n}\right)=$ $\left(y^{\prime} y\right) a_{n}=0$, and so $a_{n}=0$, a contradiction. Therefore $\left(y a_{n}\right) x_{1} \neq 0$. Now $0 \neq$ $y\left(a_{n} x_{1}\right) \in\left(e_{1} R e_{n}\right)\left(e_{n} R e_{1}\right) \subseteq e_{1} R e_{1}$. Furthermore, $y a_{n} x_{n} \in e_{1} R e_{n}$, and $a_{n} \in$ $e_{n} R e_{1}$ yield $y a_{n} x_{n} a_{n} \in e_{1} R e_{1}$. Next, multiplying (2) on the left by $y a_{n}$ we get $\left(y a_{n} x_{1}\right) a_{1}=y a_{n}-y a_{n} x_{n} a_{n}$. Consequently, $a_{1}=\left(y a_{n} x_{1}\right)^{-1}\left(y a_{n}-y a_{n} x_{n} a_{n}\right)$. This shows that $e_{1} R e_{1}$ is a left Ore domain. Similarly, we conclude that every $e_{i} R e_{i}$ is a left Ore domain.

Therefore, each $R e_{i}$ is a uniform left ideal of $R$. This is folklore; however we provide an argument here for the sake of completeness: If $A, B$ are nonzero submodules of $R e_{i}$ such that $A \cap B=0$, then $e_{i} A \cap e_{i} B=0$. Since $e_{i} A$ and $e_{i} B$ are left ideals of the left Ore domain $e_{i} R e_{i}$, either $e_{i} A=0$ or $e_{i} B=0$. Consequently, either $B A=0$ or $A B=0$. This is a contradiction because $R$ is a prime ring, proving the claim. Since $R=R e_{1} \oplus \cdots \oplus R e_{n},{ }_{R} R$ has finite uniform dimension. Moreover, as $R$ is prime right Goldie, it has DCC on right annihilators (cf. [4, Lemma 7.2.2]). Therefore, $R$ has ACC on left annihilators, proving that $R$ is left Goldie.

Finally, we show that $R$ is left CS. Note that $Q$ is the classical left and right quotient ring of $R$. Let $U$ be a non-essential left ideal of $R$. Then there are orthogonal idempotents $e, f \in Q$, such that $Q=Q e \oplus Q f$, where $U$ is essential in ${ }_{R} Q e$, and $Q f \neq 0$. Hence $U(f Q)=0$. As $f Q \cap R \neq 0$, the right annihilator of $U$ in $R$ is nonzero. Moreover, let $0 \neq a \in R$ and $r(a)$ be the right annihilator of $a$ in $R$. Then $R=C \oplus D$, where $r(a)$ is essential in $D$. Since $a R \cong R / r(a)$ and $a R$ is a nonsingular right ideal of $R$, we must have $r(a)=D$. This shows that $a R \cong C$, and in particular that $a R$ is projective. Hence $R$ is a right p.p. ring. Thus $R$ is left CS by [1, Proposition 12.3]. The proof is complete.

Remark 1. Theorem 1 is not true, in general, if the right uniform dimension of the prime right Goldie ring is 1, since there exist right Ore domains (hence right CS) which are not left Ore (hence not left CS). For the existence of such a domain, see 3, Exercise 1, p. 101].

Remark 2. Let $R$ be a semiprime right Goldie right CS ring. Then $R_{R}$ is a direct sum of uniform right ideals $e_{i} R, i=1, \cdots, n, e_{i}^{2}=e_{i}$. After renumbering the indices, if necessary, we get $R=\left[e_{1} R\right] \oplus \cdots \oplus\left[e_{t} R\right]$, where each $\left[e_{j} R\right]$ is a direct sum of uniform right ideals belonging to $\left\{e_{i} R\right\}_{i=1}^{n}$ that are subisomorphic to each other, and $\operatorname{Hom}_{R}\left(e_{j} R, e_{k} R\right)=0$ for $j \neq k(j, k \in\{1, \cdots, t\})$. It is easy to check that each $R_{j}=\left[e_{j} R\right]$ is an ideal of $R$, and is itself a prime right Goldie right CS ring. Hence $R=R_{1} \oplus \cdots \oplus R_{t}$ is a ring direct sum of prime right Goldie right 
CS rings. Let $n_{j}$ be the right uniform dimension of $R_{j}$. By Theorem 1, for any $n_{j}>1, R_{j}$ is also left Goldie and left CS.

The following consequence of Theorem 1 is a stronger version of [1. Corollary 12.9].

Corollary 2. For a domain $K$ the following conditions are equivalent:

(a) $(K \oplus K)_{K}$ is $C S$;

(b) ${ }_{K}(K \oplus K)$ is $C S$.

If $K$ satisfies (a) or (b), then $K$ is right and left Ore.

Proof. $(a) \Rightarrow(b)$. By $(a), K$ is right Ore; hence the $2 \times 2$ matrix ring $M_{2}(K)$ over $K$ is a prime right Goldie ring of right uniform dimension 2. Moreover, by [1, Lemma 12.8], (a) implies that $M_{2}(K)$ is right CS. By Theorem 1, $M_{2}(K)$ is left CS. Again by [1, Lemma 12.8], $K(K \oplus K)$ is CS, proving $(b)$. Similarly $(b) \Rightarrow(a)$ holds. The last statement is clear.

Theorem 3. For a semiprime ring $R$, the following conditions are equivalent:

(i) $R$ is left Goldie, right CS;

(ii) $R$ is right Goldie, left $C S$.

In this case, $R=R_{1} \oplus \cdots \oplus R_{n}$, where each $R_{i}$ is prime, right Goldie, left

Goldie, right $C S$ and left $C S$.

Proof. We need only show $(i) \Rightarrow(i i)$; then the implication $(i i) \Rightarrow(i)$ is obtained in a similar way.

Let $R$ be a semiprime left Goldie right CS ring. We claim that $R$ has finite right uniform dimension. Assume on the contrary, that $R$ contains an infinite direct sum $\bigoplus_{i=1}^{\infty} A_{i}$ of nonzero right ideals $A_{i}$. Let $K_{1}$ be the complement of $A_{1}$ in $R$ containing $\bigoplus_{i=2}^{\infty} A_{i}$. Since $R$ is right CS, $R=K_{1} \oplus B_{1}$ for some nonzero right ideal $B_{1}$ of $R$. Let $K_{2}$ be the complement of $A_{2}$ in $K_{1}$ containing $\bigoplus_{i=3}^{\infty} A_{i}$. Since $\left(K_{1}\right)_{R}$ is CS, $K_{1}=K_{2} \oplus B_{2}$ for some nonzero submodule $B_{2}$ of $K_{1}$. This yields $R=K_{2} \oplus B_{1} \oplus B_{2}$. Proceeding in this way we can produce an arbitrary number of orthogonal idempotents in $R$, a contradiction, because $R$ is left Goldie. Hence $R$ has finite right uniform dimension. Since $R$ is semiprime left Goldie, $R$ has DCC on left annihilators, and so $R$ is right Goldie. By Remark $2, R=R_{1} \oplus \cdots \oplus R_{t}$, a direct sum of prime right and left Goldie right CS rings. Let $n_{i}=\mathrm{u}-\operatorname{dim}\left(R_{i}\right)_{R_{i}}$ $=\mathrm{u}$-dim $\left(R_{i} R_{i}\right)$. If $n_{i}=1$, then $R_{i}$ is a uniform left $R_{i}$-module, and hence left CS. For $n_{i} \geq 2$ we apply Theorem 1 to obtain that $R_{i}$ is also left CS. Hence $R$ is left CS. The last statement is clear from the proof.

\section{ACKNOWLEDGMENTS}

Dinh Van Huynh wishes to thank the Departmant of Mathematics, Ohio University for support and hospitality during his visit in the academic years 1997-98 and 1998-99. Sergio R. López-Permouth gratefully acknowledges the support of Ohio University through a Baker Fund Award for this project.

We thank the referee for his helpful suggestions and comments.

\section{REFERENCES}

[1] N.V. Dung, D.V. Huynh, P.F. Smith and R. Wisbauer, Extending Modules, Research Notices in Mathematics Series 313, Pitman, London (1994). MR 96f:16008

[2] C. Faith, Algebra I: Rings Modules, and Categories of Modules, Springer-Verlag, BerlinHeidelberg-New York 1981. MR 82g:16001 
[3] K.R. Goodearl, Ring Theory: Nonsingular Rings and Modules, Marcel Dekker, New YorkBasel 1968. MR 55:2970

[4] I.N. Herstein, Noncommutative Rings, The Carus Mathematical Monograph No 15, Math. Ass. Amer. 1973. $\mid$ MR 37:2790

Institute of Mathematics, P.O. Box 631 Boho, Hanoi, Vietnam - Department of Mathematics, Ohio University, Athens, Ohio 45701

Department of Mathematics, Ohio University, Athens, Ohio 45701

Department of Mathematics, Ohio University, Athens, Оhio 45701 